\title{
Precise determination of the muon reconstruction efficiency in ATLAS at Run-II
}

\author{
Despoina Sampsonidou, ${ }^{1, a}$ on behalf of the ATLAS Collaboration \\ ${ }^{1}$ Aristotle University of Thessaloniki
}

\begin{abstract}
In Run-2 of the LHC, the ATLAS experiment reconstruction algorithm has been improved and extended compared to the one used in Run-1. In this presentation, we will discuss the precise measurement of the muon reconstruction efficiency measured in pp collisions at $\sqrt{(s)}=13 \mathrm{TeV}$ in 2015 and 2016 using samples of $J / \psi \rightarrow \mu \mu$ and $Z \rightarrow \mu \mu$ decays. The reconstruction efficiency is measured using different methods in the various regions of the detector and for muon momenta between 6 and hundreds of $\mathrm{GeV}$.
\end{abstract}

\section{Introduction}

These proceedings report on the precise measurement of the muon reconstruction efficiency measured in pp collisions at sqrt(s) $=13 \mathrm{TeV}$ in 2015 using samples of $J / \psi \rightarrow \mu \mu$ and $Z \rightarrow \mu \mu$ decays. The reconstruction efficiency is measured using different methods in the various regions of the detector and for muon momenta between 6 and hundreds of GeV. Precise determination of the reconstruction efficiency of muons is essential in particle physics to accurately test Standard Model [2-4]processes or the search for processes beyond the Standard Model[5, 6].

\section{The ATLAS Detector}

A full description of the ATLAS detector can be found in Ref. [1]. Muons are identified and reconstructed by exploiting information from the Inner Detector (ID) and the Muon Spectrometer (MS) and to a lesser extent the calorimeters. The ID is the detector closest to the interaction point. It is designed to provide high precision tracking and high resolution momentum measurements for charged particles, covering pseudorapidity of $|\eta|<2.5$. It uses magnetic field of $2 \mathrm{~T}$ and consists of three subdetectors: 4 layers of pixel sensors and 4 layers of silicon strips with a pseudorapidity coverage up to 2.5 , and 72 straw layers of Transition Radiation Tracker (TRT) with a pseudorapidity range up to 2.0 .

The MS forms the outer part of the ATLAS detector. It provides precise measurement of muon momenta up to several $\mathrm{TeV}$, covering pseudorapidity range of $|\eta|<2.7$, and trigger coverage in the pseudo rapidity range of $|\eta|<2.4$. The MS includes also magnetic field provided by three toroid magnet systems. An air-core toroid in the barrel region $0<|\eta|<1.6$ and two toroids in the end

ae-mail: despoina.sampsonidou@cern.ch 


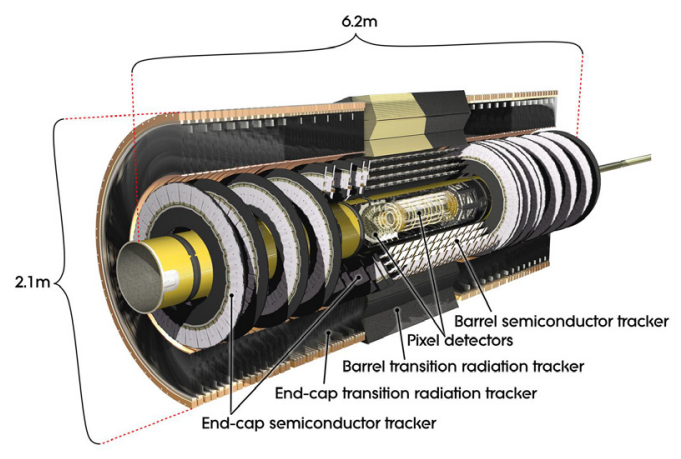

Figure 1. The Inner Detector

caps region of $1.4<|\eta|<2.7$ provide a mean value of $0.5 \mathrm{~T}$, allowing for stand-alone momentum measurement in principle without the need of combining the MS and the ID track reconstruction [9]. The MS consists of a barrel and two endcap sections, with three stations of trigger chambers, Resistive Plate Chambers (RPC) in the barrel and Thin Gap Chambers (TGC) in the end-caps and three stations of precision chambers, Monitored Drift Tube chambers (MDT). The innermost station in the endcaps was replaced by Cathode Strip Chambers (CSC).

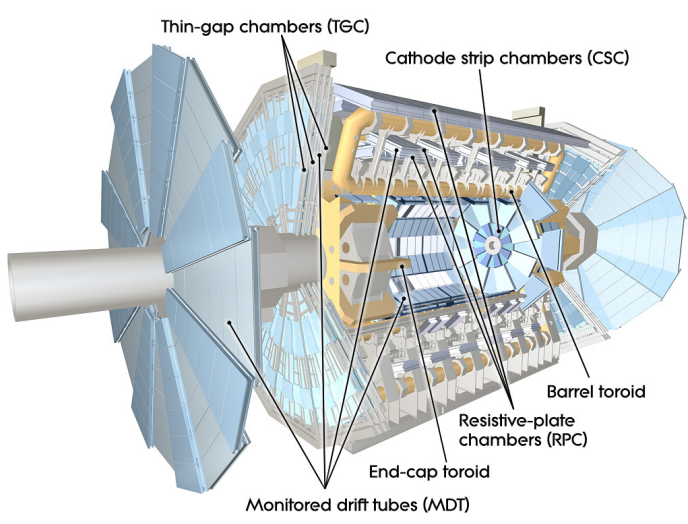

Figure 2. The Muon Spectrometer

\section{Muon Identification}

Muon identification is performed by combining information from the MS, the ID and the calorimeter. Reconstructed muons can be grouped into four different complementary categories depending on the sub detector systems used in the reconstruction: 
Stand-alone muons (MS): muon tracks that are reconstructed only in the MS and are extrapolated to the beam line accounting for energy losses. The MS magnetic field allows for stand-alone momentum measurements up to $|\eta|<2$.7.

Combined muons (CB): They are muon tracks that are reconstructed in the MS and are associated to ID tracks. The best purity and momentum resolution is provided by the combination of the information from both detectors.

Segment-tagged muons: They are ID tracks which are extrapolated to the MS. These tracks are usually associated to track segments in the first layer of the MS that were not used in the reconstruction of a full track in the MS. The segment-tagged muons improve the total reconstruction efficiency since they correspond to low-pT muons which do not reach the middle and outer stations due to bending effects of the magnetic field.

Calorimeter-tagged muons: They are ID tracks which are reconstructed in a region with no coverage from the MS. This is a small region around $\eta=0.0(|\eta|<0.1)$. ID tracks associated with an energy deposit in the calorimeter which is consistent with a minimum ionizing particle, are considered as muon candidates. These muons are used to recover the acceptance loss in the non-instrumented region of the MS, even though they have the lowest purity of all types of muons.

\section{Muon Reconstruction Efficiency}

Depending on the kinematics and desired purity, the muons form four identification selections:

Loose muons: The Loose identification criteria are designed to maximise the reconstruction efficiency while providing good-quality muon tracks. They are specifically optimised for reconstructing Higgs boson candidates in the four-lepton final state. All muon types are used.

Medium muons: This selection minimises the systematic uncertainties associated with muon reconstruction and calibration. Only CB and MS muons are used.

Tight muons: Tight muons are selected to maximise the purity of muons at the cost of some loss in efficiency. Only CB muons with hits in at least two stations of the MS and satisfying the Medium selection criteria are considered.

High- $p_{T}$ muons: The High-pT selection aims to maximise the momentum resolution for muons with transverse momentum above $100 \mathrm{GeV}$. The selection is optimised for searches for high-mass $Z^{\prime}$ and $W^{\prime}$ resonances $[8,9]$. CB muons passing the medium selection and having at least three hits in three MS stations are selected.

In the region $|\eta|<2.5$, the muon reconstruction in the ID and the MS is performed independently, a precise determination of the muon reconstruction efficiency is obtained by using the tag-and-probe method. In the region $2.5<|\eta|<2.7$, the reconstruction is performed solely in the MS, so a different methodology is used to estimate the efficiency in this eta region. 


\subsection{Reconstruction Efficiency for $|\eta|<2.5$}

The reconstruction efficiency is measured using a tag-and-probe method on $Z \rightarrow \mu \mu$ and $J / \psi \rightarrow \mu \mu$ events [7]. A selection of an almost pure muon sample from $Z \rightarrow \mu \mu$ and $J / \psi \rightarrow \mu \mu$ events is made. The $\mathrm{Z}$ decays provide a sample of probes with $p_{T}>10 \mathrm{GeV}$ and the $J / \psi$ decays provide a sample of 5 $\mathrm{GeV}<p_{T}<15 \mathrm{GeV}$. A leg of the decay is required to be a medium muons that triggers the event (tag). The second leg of the decay is required to be an ID track or an Id track with calorimeter tagging (if the reconstruction efficiency of the MS is measured) or an MS track (if the reconstruction efficiency of the ID is measured). The fraction of probe tracks that were successfully reconstructed as muons measures the efficiency. In order for a track to be considered as successfully reconstructed muon, a reconstructed muon is required to be found within a cone in the $\eta-\phi$ plane of size $\Delta R=\sqrt{\Delta \eta^{2}+\Delta \phi^{2}}<0.05$ around the probe track. The efficiency measurement is carried out in data and simulation and a scale factor is computed as the ratio of the two. The scale factors are applied to the MC in order to correct for a possible mismodelling of the muon reconstruction efficiency. In Fig. 3,4 the measured muon reconstruction efficiencies for varying $\eta$ for medium and high- $p_{T}$ muons from $Z \rightarrow \mu \mu$ samples are presented whereas in Fig. 5 the muon reconstruction efficiencies with varying $\eta$ for loose muons from $J / \psi \rightarrow \mu \mu$ is presented. In Fig. 6, the muon reconstruction efficiency as a function of transverse momentum is presented, with measurements from both $Z \rightarrow \mu \mu$ and $J / \psi \rightarrow \mu \mu$ events.

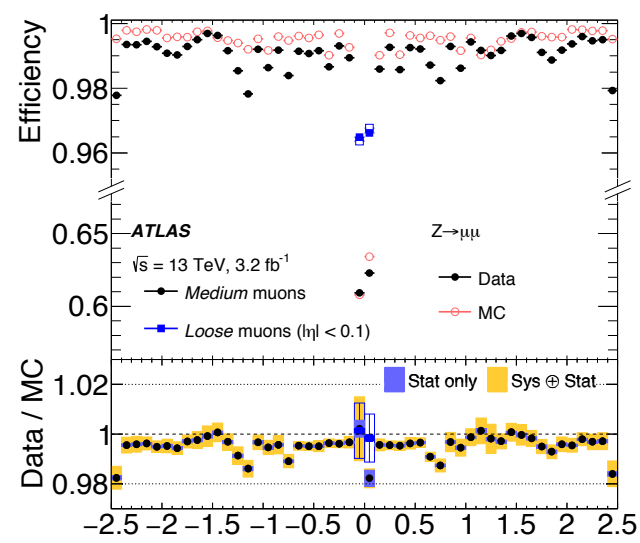

$\eta$

Figure 3. Measured reconstruction efficiency for varying eta of the ATLAS muon spectrometer for medium muons, from $Z \rightarrow \mu \mu$. The panel at the bottom shows the ratio of the measured to predicted efficiencies, with statistical and systematic uncertainties. Taken from Ref. [11] .

\subsection{Reconstruction Efficiency for $|\eta|>2.5$}

The ID acceptance restricts the reconstruction of the combined muons to pseudorapidity region $|\eta|<$ 2.5. For $2.5<|\eta|<2.7$, a measurement of the efficiency SF is performed by using the double ratio method. The number of muons observed in $Z \rightarrow \mu \mu$ decays in the high eta region is normalized to the number of muons observed in the region $2.2<|\eta|<2.5$, both for data and simulation [10]. The high eta scale factor is derived as the ratio of the two results and it is presented in Fig. 7, as a function of the detector phi. 


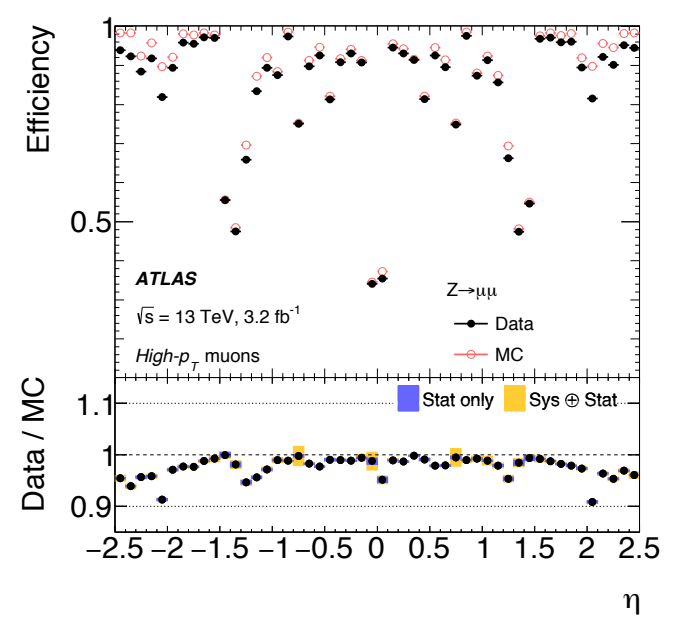

Figure 4. Measured reconstruction efficiency for varying eta of the ATLAS muon spectrometer for High- $p_{T}$ muons, from $Z \rightarrow \mu \mu$ sample. The panel at the bottom shows the ratio of the measured to predicted efficiencies, with statistical and systematic uncertainties. Taken from Ref. [11] .

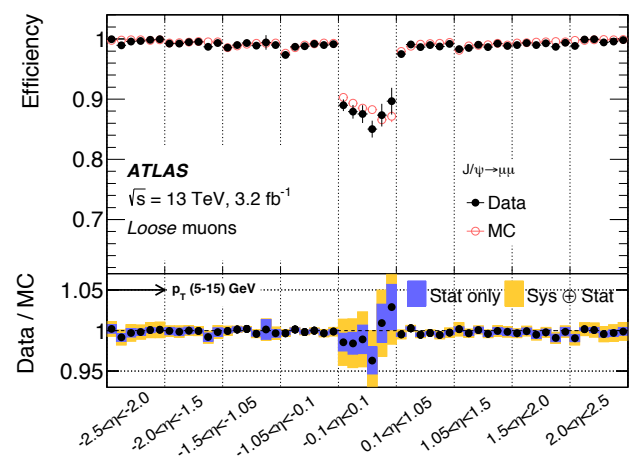

Figure 5. Measured reconstruction efficiency for varying eta of the ATLAS muon spectrometer for Loose muons with $5 \mathrm{GeV}<p_{T}<15 \mathrm{GeV}$, from $J / \psi \rightarrow \mu \mu$ sample.The panel at the bottom shows the ratio of the measured to predicted efficiencies, with statistical and systematic uncertainties. Taken from Ref. [11].

\section{Conclusion}

These proceedings summarized the determination of the muon reconstruction efficiency in the various regions of the detector, and for muon momenta between 6 and hundreds of GeV. The reconstruction efficiency is found to be in good agreement with the simulation. 


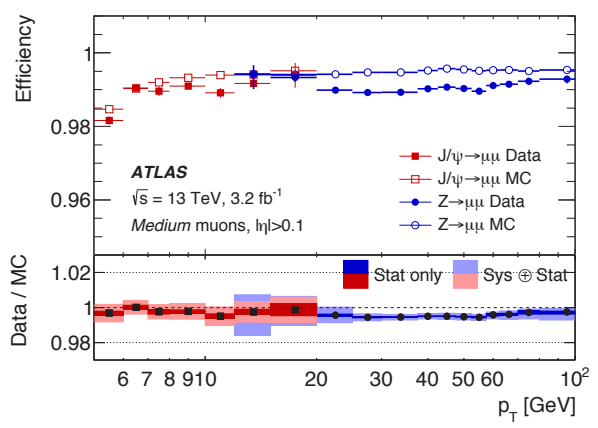

Figure 6. Muon reconstruction efficiency as a function of the transverse momentum. The panel at the bottom shows the ratio of the measured to predicted efficiencies, with statistical and systematic uncertainties. Taken from Ref. [11] .

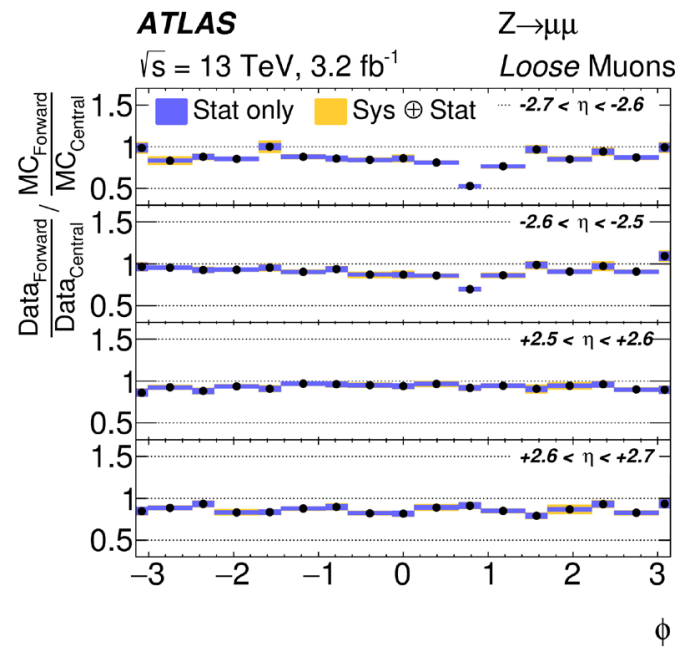

Figure 7. Double ratio for the forward muons for varying phi. Taking into account all corrections to the simulation for the central pseudorapidity region, this quantity measures the reconstruction efficiency scale factor for the forward region, where the standard tag-and-probe technique cannot be used due to missing coverage by the Inner Detector. Taken from Ref. [11] .

\section{References}

[1] ATLAS Collaboration, JINST 3, S08003 (2008).

[2] ATLAS Collaboration, Phys. Rev. D 85, 072004 (2012). arXiv:1109.5141 [hep-ex]

[3] ATLAS Collaboration, JHEP 1406, 112 (2014). arXiv:1404.1212 [hep-ex]

[4] ATLAS Collaboration, JHEP 1405, 068 (2014). arXiv:1402.6263 [hep-ex]

[5] ATLAS Collaboration, Phys. Rev. D 87, 015010 (2013). arXiv:1211.1150 [hep-ex]

[6] ATLAS Collaboration, Phys. Rev. D 88, 072001 (2013). arXiv:1308.4075 [hep-ex] 
[7] ATLAS Collaboration, Eur. Phys. J. C (2016). arXiv:1603.05598 [hep-ex]

[8] ATLAS Collaboration, ATL-PHYS-PUB-2015-037 (2015),http://atlas.web.cern.ch/Atlas/GROUPS/PHYSIC 2015-10/

[9] ATLAS Collaboration, The ATLAS Experiment at the CERN Large Hadron Collider, JINST 3 (2008) S08003

[10] ATLAS Collaboration,Eur.Phys.J. C74 (2014)

[11] Eur. Phys. J. C (2016) 76:292 